\title{
INKJET PRINTED COLORIMETRIC AMMONIA SENSOR ON PLASTIC FOIL FOR LOW-COST AND LOW-POWER DEVICES
}

\author{
Jerome Courbat $^{1}$, Michael Linder ${ }^{1}$, Maxime Dottori ${ }^{1}$, Danick Briand ${ }^{1}$, \\ Jürgen Wöllenstein ${ }^{2}$, Nico F. de Rooij ${ }^{1}$ \\ ${ }^{1}$ Ecole Polytechnique Fédérale de Lausanne (EPFL), \\ Institute of Microengineering, Neuchâtel, Switzerland \\ ${ }^{2}$ Fraunhofer Institute IPM, Freiburg im Breisgau, Germany
}

\begin{abstract}
A polymeric-based colorimetric gas sensor with its associated electronics targeting ammonia detection at low-cost and low-power is presented. The gas sensitive layer was inkjet printed on a plastic foil used as an optical waveguide. The planar configuration of the sensor makes it compatible with large scale fabrication techniques, such as rollto-roll processes. Its power consumption and noise level were measured. The sensor exhibited a good sensitivity to ammonia with a theoretical limit of detection of $104 \mathrm{ppb}$ in a constant operation mode. The power consumption was reduced to the sub$\mathrm{mW}$ range when operating in pulsed mode. It foresees applications in the field of wireless systems, for environmental and safety monitoring.
\end{abstract}

\section{INTRODUCTION}

Optical gas sensors have recently gained more interest since they can provide a very good sensitivity and selectivity at low-cost. Among them, colorimetric gas sensors offer a good alternative in combining simplicity, low-power operation and cost effectiveness [1,2].

The author previously presented the development of colorimetric films for the detection of ammonia in a waveguide configuration $\left(\mathrm{NH}_{3}\right)$ [3]. These films were based on a $\mathrm{pH}$ indicator embedded in a polymeric matrix and subsequently spin-coated onto glass slide and coupled to external LED and photo-detector components.

In this communication, we report on a low-cost and low-power colorimetric gas sensor based on plastic technologies and additive processes targeting applications in autonomous and wireless systems. It is based on plastic foil - PEN or PET - as substrate and directly used as a planar optical waveguide to simplify its fabrication. Additionally to the cost effectiveness of the materials compared to glass, it brings several advantages such as being compatible with large scale fabrication techniques, e.g. roll-toroll processes. Instead of using spin-coating, the chemochromic film sensitive to $\mathrm{NH}_{3}$ was deposited directly and locally on the substrate by inkjet printing, an additive deposition method compatible with printed electronics processing technologies.

Moreover, the colorimetric gas sensor was combined with SMD optoelectronic components and a custom-made ultra-low power driving and readout circuitry to target low-power applications such as wireless systems. It exhibited a theoretical limit of detection (LOD) of $\mathrm{NH}_{3}$ of $104 \mathrm{ppb}$ in its present configuration when operating in continuous mode. The power consumption of the whole system was reduced to the sub-mW range by operating it in a pulsed mode at a cost of a higher limit of detection.

\section{EXPERIMENTAL}

\section{Principle of operation}

A cross-sectional view of the ammonia gas sensor on plastic substrate is depicted in figure 1. It consisted in the detection of the color change of a chemochromic film sensitive to a specific gas such as $\mathrm{NH}_{3}$. The gas sensitive film laid on a planar optical waveguide made of a plastic foil. SMD optoelectronic components were used as light source and detectors. The light propagates in the waveguide by total internal reflection due to its higher refractive index than the surrounding media. The light coupling between the light source, the optical waveguide and the detectors - one as a reference and one for the measurement - was guaranteed by micro-mirrors shaped on the plastic foil. A change in light intensity reaching the measurement detector is observed when the gas sensitive film changes its color when exposed to the gas to be detected.

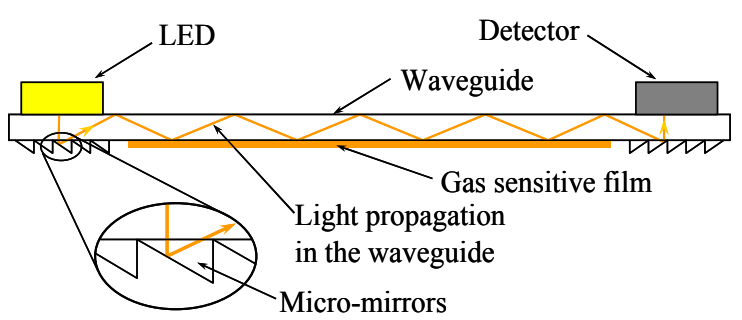

Fig. 1: Cross-sectional schematic of the gas sensor. 


\section{Fabrication of the colorimetric sensor}

Optically clear waveguides were cut out of 125 and $200 \mu \mathrm{m}$ thick PEN (Teonex Q65FA from Dupont) or 125 and $250 \mu \mathrm{m}$ thick PET foils (Melinex 506 from Dupont). The influence of the length of the waveguide on the light losses and the gas response was investigated by designing waveguides with two different lengths, 23 and $46 \mathrm{~mm}$. Due to the nature of the materials involved, their fabrication was based on low temperature processing. Micromirrors were patterned with a PDMS mold in a UVcurable optically clear epoxy resist. The influence of a metal layer covering the mirrors on the optical losses and gas response was investigated by e-beam evaporating a $300 \mathrm{~nm}$-thick aluminum layer to achieve a proper step covered edge. A more detailed description of the fabrication of the polymeric micro-mirrors can be found in [4].

The chemochromic layer was composed of BPB (bromophenol blue) as sensitive element to $\mathrm{NH}_{3}$ embedded in polymeric matrix made of PMMA and a hydrophobic plasticizer (tributyl phosphate) [3]. The choice of solvents for their dissolution was based on the ink specifications required by the inkjet printer used (DMP-2831 from Dimatix). Moreover, solvent with a high boiling point were preferred to reduce coffee ring effect with the ink, which is due to a too fast evaporation of the solvents (fig. 3). Between one and ten layers of the colorimetric film were inkjet printed to reach different thicknesses from 200 to $600 \mathrm{~nm}$. This additive deposition technique permits a very precise localization of the colorimetric film along the measurement channel compared to spin-coating [4]. Finally, the SMD photonic components, the light source and the detectors, were glued (fig. 4).
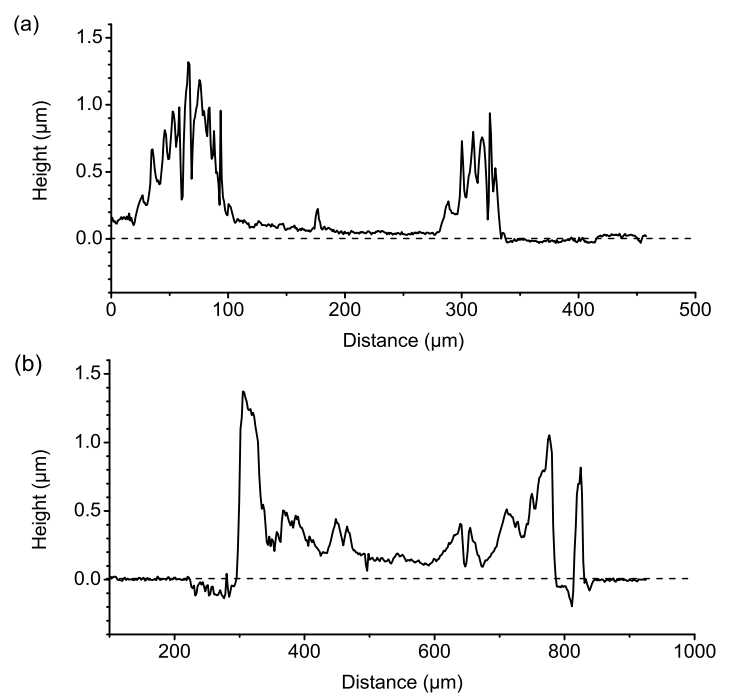

Fig. 3: Profile of one printed layer of the colorimetric film dissolved in a solvent with (a) low and (b) high boiling point.

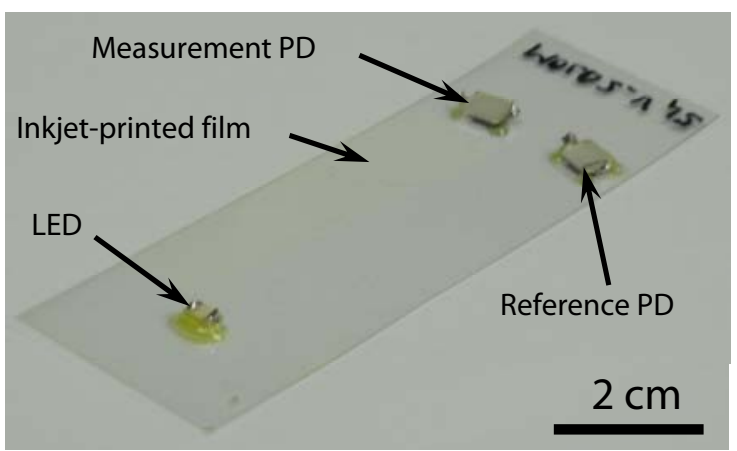

Fig. 4: Optical picture of the colorimetric ammonia sensor.

\section{Characterization of the waveguides}

The influence of each parameter of the waveguide on the optical losses and gas response - material, thickness, length and mirrors type (with or without $\mathrm{Al}$ ) - were investigated and analyzed through full or partial fractional factorial analysis.

Optical losses were measured in a black box with the same LED and photodiodes used for the actual gas measurements. The latter were performed in nitrogen as gas carrier with $50 \%$ of relative humidity with a flow of $500 \mathrm{sccm}$. The sensors were exposed to concentrations of $\mathrm{NH}_{3}$ ranging from $20 \mathrm{ppm}$ down to $200 \mathrm{ppb}$.

The evaluation of the sensors was carried out in three steps with the electronic circuitry operating in continuous mode. First, the design of the waveguide on their gas sensing abilities was investigated when ten layers of inkjet printed colorimetric film were deposited to achieve a good sensitivity of the sensors. Once the best waveguide configuration was determined, the influence of the thickness of the inkjet printed gas sensitive film was investigated. 1, 3, 5 and 10 layers of colorimetric film were deposited and gas measurements were performed. Finally, once the best combination of the transducer and the thickness of the gas sensitive layer were defined, the gas sensor was tested with the electronic circuitry operating in different modes. Their power consumptions and noise level were measured and the limit of detection of $\mathrm{NH}_{3}$ was evaluated using the $3 \sigma$ method [3].

\section{Electronic driving and readout circuitry}

An electronic driving and readout circuitry designed from ultra-low power components was implemented to operate the sensor (fig. 5). It was based on ultra-low power MSP430 microcontroller from Texas Instruments combined with a yellow LED (TLYK1100C from Toshiba) as light source since the chemochromic film exhibited the highest variation of light absorption at such wavelengths. 


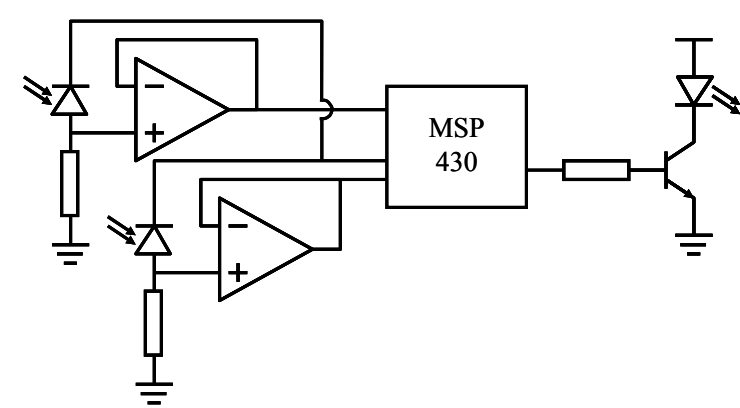

Fig. 5: Electronic driving and readout circuitry with a LED as light source and with photodiodes as detectors.

The response of the sensor came from the difference of the signal measured by two photodiodes (BPW34S from Osram), one reference and one for the gas measurement. They were coupled to two very low power operational amplifiers (OPA2369 from Texas Instrument). A feedback loop allowed keeping the light intensity of the LED constant over time. This circuitry was used in both constant mode - LED always on - and pulsed mode - with the LED turned on for 3 and $9 \mathrm{~ms}$ every second - of operation. These periods showed to be reasonable to regulate the LED.

\section{RESULTS AND DISCUSSION}

\section{Optical losses in the waveguides}

Figure 6 presents the optical losses for all the waveguides investigated. Short and thick waveguides exhibited the lowest attenuation. The losses were drastically increased by the coating of the mirrors. Besides that factor, an analysis of variance found that the length of the waveguide had the major influence on the optical losses and should be kept in a short configuration. The type of substrate and its thickness had only a small impact on the light attenuation.

\section{Gas measurements}

A typical response of the colorimetric gas sensor when exposed to $\mathrm{NH}_{3}$ is presented in figure 7. A drawback of such sensors is there low response time. However, the kinetics of the chemical reaction was improved by heating up the transducer at $40^{\circ} \mathrm{C}$ and $60^{\circ} \mathrm{C}$ reducing the recovery times, $t_{90 \%}$, in the order of the hour at room temperature was reduced to few minutes. Moreover, the inkjet printed film exhibited a slightly longer response time than the one obtained with the spin-coated film [4]. Its origin might come from the different of solvent used for both techniques of deposition and/or the irregular thickness of the film obtained when inkjet printed, leading to a longer diffusion within the

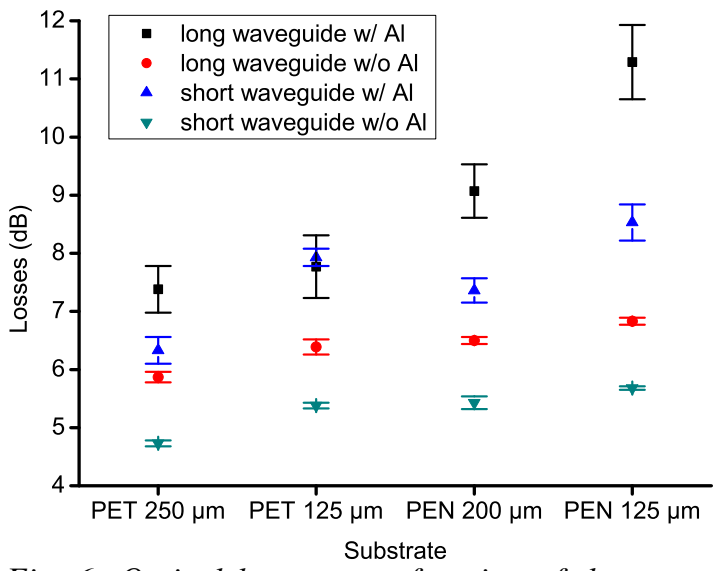

Fig. 6: Optical losses as a function of the type of waveguide. Confidence interval: $95 \%$.

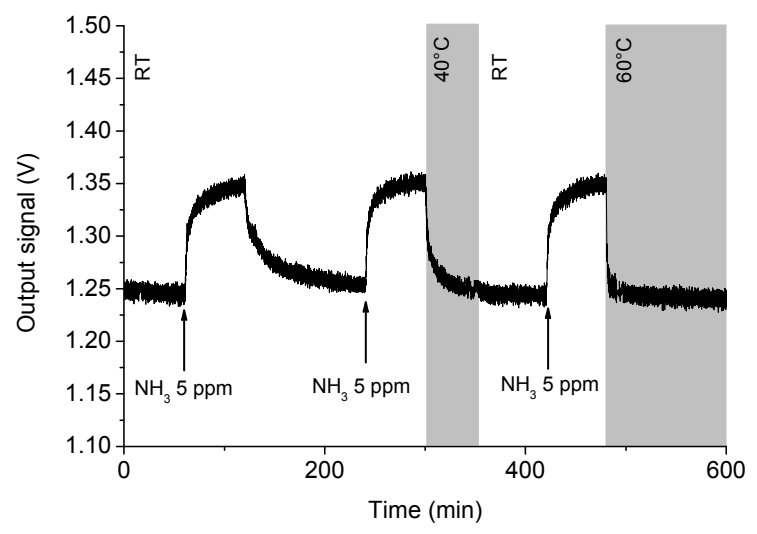

Fig. 7: Response of the sensor when expose to 5 ppm of $\mathrm{NH}_{3}$ in synthetic air with $50 \% \mathrm{RH}$, gas flow of $500 \mathrm{ml} / \mathrm{min}$. The desorption rate was increased by heating up the waveguide.

polymeric matrix, prolonging thus the kinetic of the chemical reaction between $\mathrm{NH}_{3}$ and the dye.

Figure 8 presents the results of a fractional factorial design of waveguides when exposed to $5 \mathrm{ppm}$ of $\mathrm{NH}_{3}$. Short waveguides gave the highest gas response. The best waveguide was based on a short, $125 \mu \mathrm{m}$-thick PEN foil with uncovered mirrors. An analysis of variance revealed the length of the waveguide had the highest influence on the gas response and should be thus kept in a short configuration. For the long waveguides, the higher optical losses might reduce the sensitivity of the gas sensor. The thickness of the foil had only a reduced impact on the sensor's response as well as the coating of the mirrors. The influence of the material of the waveguide was intermediate.

The thickness of the colorimetric film on the sensor response was also investigated. Thicker films gave a higher response to $\mathrm{NH}_{3}$ (fig. 9). Their thickness from 200 to $600 \mathrm{~nm}$ - was difficult to be properly measured due to the high surface roughness of the film deposited (fig. 3). A tradeoff has to be found between solvents with a low boiling point leading 


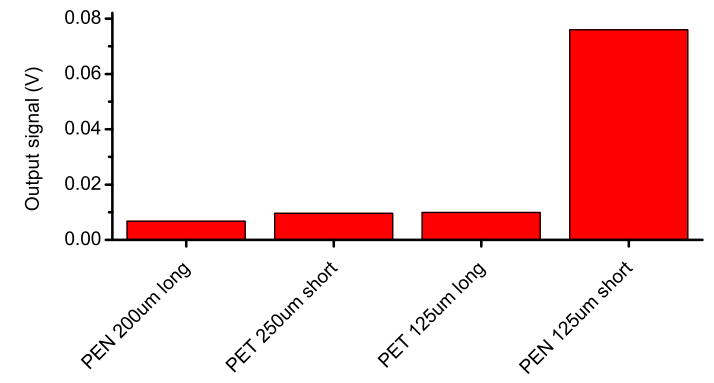

Fig. 8: Gas response of waveguides to 5 ppm of $\mathrm{NH}_{3}$. Short and thin PEN waveguides without covered mirrors gave the highest response.

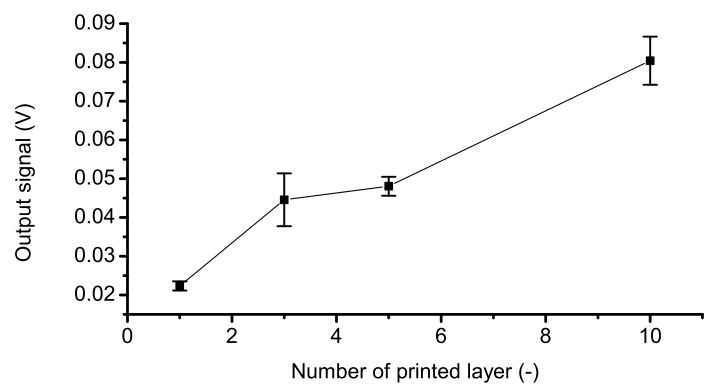

Fig. 9: Response to 5 ppm of $\mathrm{NH}_{3}$ as a function of the number of printed layer. Conf. interval: $95 \%$.

to coffee ring effect, and a solution with a higher boiling point involving a rougher and uneven surface. Thus, ten layers of colorimetric film deposited on short, $125 \mu \mathrm{m}$ thick PEN waveguides were used for the evaluation of the electronic circuitry.

\section{Electronic readout circuitry}

The electronic circuitry was evaluated in terms of power consumption, sensitivity and noise level (fig. 10). The most power consuming device of the circuitry was the yellow LED. Its operation in a pulsed mode reduced the power consumption of the system from $8 \mathrm{~mW}$ to $868 \mu \mathrm{W}$, increased the noise level from 3 to $33 \mathrm{mV}$ and the limit of detection

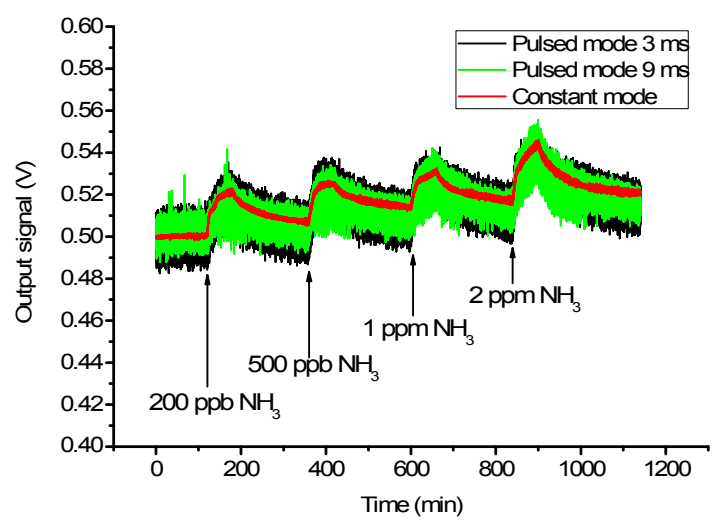

Fig. 10: Gas response to NH3 with the electronic circuitry operating in constant and pulsed modes. from $104 \mathrm{ppb}$ to $1.4 \mathrm{ppm}$ (Table 1). The latter can be reduced by increasing the regulation time of the light source at the cost of a higher power consumption. The best compromise was the circuitry operating in pulsed mode with the LED turned on for $9 \mathrm{~ms}$ (duty cycle of $0.9 \%$ ) for a power consumption of $947 \mu \mathrm{W}$.

Table 1. Influence of the operation mode on the sensing abilities of the $\mathrm{NH}_{3}$ sensor.

\begin{tabular}{llll}
\hline $\begin{array}{l}\text { Mode of } \\
\text { operation }\end{array}$ & $\begin{array}{l}\text { Power cons. } \\
{[\mu \mathrm{W}]}\end{array}$ & $\begin{array}{l}\text { Noise } \\
{[\mathrm{mV}]}\end{array}$ & $\begin{array}{l}\text { LOD } \\
{[\mathrm{ppb}]}\end{array}$ \\
\hline Continuous & 7980 & 3 & 104 \\
Pulsed 9 ms & 947 & 20 & 800 \\
Pulsed 3 ms & 868 & 33 & 1400 \\
\hline
\end{tabular}

\section{CONCLUSION}

With the printing of the colorimetric film and patterning of polymeric micro-mirrors on plastic foil, a major step was achieved towards the implementation of full plastic selective gas sensors. The highest gas response was obtained with a short and thin PEN waveguide with uncoated mirrors. The presented system exhibited a power consumption of $947 \mu \mathrm{W}$ in a pulsed mode of operation for a limit of detection of $\mathrm{NH}_{3}$ of $800 \mathrm{ppb}$. The combination with commercial OLED and PPD would further lead to an integrated all polymeric optical transducer on plastic foil fully compatible with printed electronics processes.

\section{ACKOWLEDGEMENTS}

We would like to thank the GOSPEL European Network of Excellence on "Artificial Olfaction and Advanced Gas Sensing Technologies" for partially funding this project. We are also grateful the Division-C of the CSEM for the metallization of the micro-mirrors.

\section{REFERENCES}

[1] R.L. Shepherd, W.S. Yerazunis, K.T. Lau, D. Diamond, Low-cost surface mount LED gas sensor, IEEE Sensors Journal, 6(4) (2006) pp. 861-866.

[2] T. Mayr et al., A novel sensor concept paving the way for the integration of OLEDs and organic photodiodes. Proc. of the Eurosensors XXII Conference, Dresden, Germany, Sept. 7-10, 2008, pp. 1300-1303.

[3] J. Courbat et al., Evaluation of $\mathrm{pH}$ indicatorbased colorimetric films for ammonia detection using optical waveguides, Sensors and Actuators B 143 (2009) 62-70.

[4] J. Courbat et al., Colorimetric gas sensors based on optical waveguides made on plastic foil, Procedia Chemistry, 1(1)(2009), pp. 576-579. 